\title{
Author Index Vol. 29, 1997
}

Aakre, B.M. 67 Abiko,T. 139 Aiello,L.P. 354 Akiba,J. 37 Alcoriza, N. 227 Ali,R.R. 261

Alió,J.L. 145 Aim, A. 57 Amemiya, T. 12 Andersson, L. 130 Augusteyn, R.C. 161 Aurora, Y. 124

Bagatin, J. 6 Bar-Пan,A. 110 Beilin,M. 110 Bellot,J.L. 227 Benedetti, E.L. 177 Bhattacharya, S.S. 261 Bhutto, LA. 12 Biedermann, B. 326 Blanco, A. 227 Bloemendal, H. 177 Bojic, L. 6 Borchman, D. 191 Bringmann, A. 326 Byrdwell, W.C. 191

Carpineto, P. 172 Cejková,J. 154 Cerovski, B. 6 Chen,H.-B. 48 Cho,C. 374 Ciancaglini, M. 172 Clayton, R.M. 207 Coffey,PJ. 305 Costagliola, C. 172 Cousins, S.W. 381 Crabbe, M.J.C. 421

Cray,C. 381 Crider,J.Y. 117 Cuthbert,J. 207

Denis, P. 218 Deudon-Combe, A. 290 Dix,R.D. 381

Donnelly, C.A. 207 Doughty, M.J. 67 Dreyfus, H. 290 Dunia, 1. 177

Eckmiller, R. 281 Elena, P.P. 218 Enzmann, V. 298, 326

Faude,F. 298,326 Francke, M. 326 Frank, R.N. 341 Fujihara, T. 103 Fujii, Y. 48 Funk,R.H.W. 320

Gaag, R. van der 130 Gabel,V.P. 269 Gallenga, P.E. 172 García-Cabanes, C. 227 Goode,D. 421

Graf,H.G. 269 Graw,J. 161 Green, K. 1 Griffin, B.W. 117 Guenther,E. 269 Gündüz, K. 405

Haemmerle, H. 269 Hariton, C. 227 Hayasaka, S. 365 Hayashi, H. 24 Heinemann, U. 326 Hicks, D. 290 Hikichi, T. 37 Hinton,D.R. 242 Hiraki,S. 365 Hockwin, O. 75,436 Hodges, L.C. 1

Hoefflinger, B. 269 Hofman,P. 130 Hozo, I. 6 Hunt,D.M. 261

Ishiko, S. 139 Ivanisevic, M. 6

Kado,M. 37 Kadoi,C. 365 Kakehashi, A. 37 Kato,Y. 139 Kim,J. 374 Kimura, H. 242 Kimura, M. 91 Kitaya,N. 139 Koenig,J.L. 429 Kohen,L. 241,298 Kohler, K. 269 Koji,T. 24 Kojima, M. 75 Korte,G.E. 393 Kuhrt,H. 326

Laria,C. 145 Lawrence, J.M. 305 Lee, S.-M. 83 Leverge, R. 218 Li,M.-J. 83 Liang, R.-C. 83

Lin, S.-Y. 83 Linden, C. 57 Lund,R.D. 305

Maignen, F. 218 Majima, Y. 61 Malik, P. 31,124 Mastropasqua, L. 172 Miliczek, K.-D. 269

Miyata,T. 409 Mohand-Said, S. 290 Morgante, A. 172 Mori,F. 139 Mosinger Ogilvie, J. 290

Murata,T. 242

Nagaoka,T. 139 Nakagawa, M. 409 Nakane,P. 24 Nisch, W. 269 Nordmann, J.P. 218

Ogasawara, H. 139 Ohnishi, Y. 91

\section{KARGER}

E-Mail karger@karger.ch Fax+ 41613061234 http://www. karger. ch

(C) 1997 S. Karger AC Basel

Ohno,S. 48 Ohsato, M. 24 Ohtani,O. 365 Okada,Y. 91 Ooshima, A. 91 Orts,A. 227 Oshima, K. 24 Osuský,R. 31, 124,242 Ou,B. 48 Özdemir, Ö. 405

Palmero, M. 227 Pannicke, T. 326 Petti, L. 172 Phillips, C.I. 207 Plater, M.L. 421 Pollack, A. 393 
Reggio,P.H. 1 Reichel, M.B. 261 Reichelt,W. 326 Reichenbach, A. 326 Ropers, H.H. 252

Rostène,W. 218 Ruiz-Moreno, J.M. 145 Ryan, St.J. 31,124,242

Sahel,J. 290 Saika,S. 91

Saito,K. 103 Sakamoto, T. 242 Sardelic, S. 6 Sasaki, K. 75 Sauvé, Y. 305 Schlingemann, R.O.

130 Schubert, M. 269 Schwartz, B. 42 Seth,J. 207 Sharif, N.A. 117 Shigemitsu, T. 61

Shimizu,Y. 61 Shin,B. 374 Shui, Y.B. 75 Silverman, M.S. 290 Simonutti, M. 290 Skatchkov,

S.N. 326 Spee, C. 242 Stanic, R. 6 Steely, H.Th. 177 Stett,A. 269 Stöger, T. 161 Stolzenburg, J.U. 326 Stout, T.J. 242

Takeuchi,T. 103 Tanaka,M. 409 Tanaka, S.-i. 91 Tenkova,T. 290

Terada, N. 48 Tilleul,P. 218 Tonoe,O. 91 Tran-Minh,D. 290 Troost, D. 130 Tsubota,K. 103

Tsukahara, S. 48

Ueda, H. 48 Ueno, N. 37

Vandegaer, K. 177

Weiss, S. 269

Wiedemann, P. 241, 298, 326 Williams, G.W. 117 Williams, T.R. 429 Wu,K. 75 Wysocki, A. 42 Yamanaka, O. 91

Yanagiya, N. 37 Yang,Y. 374 Yorio,T. 117 Yoshida,A. 37,139

Zink,J.M. 429 Zrenner, E. 269

Author Index

Ophthalmic Res Vol. 29, 1997

439 